\title{
Erratum: Constituent quark-light vector mesons effective couplings in a weak background magnetic field [Phys. Rev. D 97, 014022 (2018)]
}

Fábio L. Braghin®

Q (Received 13 January 2020; accepted 21 January 2020; published 11 February 2020)

DOI: $10.1103 /$ PhysRevD.101.039902

Several coupling constants of axial mesons to constituent quarks were defined incorrectly in this paper. Besides the corrected axial mesons-constituent quarks coupling constants, a few vector mesons-constituent quarks coupling constants are rewritten differently below. The numerical results presented in that paper are not modified since they were done for the vector mesons couplings.

The leading vector or axial mesons-photon couplings to constituent quarks in the long wavelength local limit, by omitting the spacetime dependencies, as presented in Eqs. (12)-(18), should be written as

$$
\begin{gathered}
I_{F F}=2 g_{1} i \epsilon_{i j 3}\left(F_{\mu \nu} \mathcal{G}_{i}^{\mu \nu} j_{p}^{j}+F_{\mu \nu} \mathcal{F}_{i}^{\mu \nu} j_{s}^{j}\right), \\
I_{F F m}=g_{1}\left[F_{\mu \nu} \mathcal{F}_{3}^{\mu \nu} j_{s}+F_{\mu \nu} \mathcal{F}^{\mu \nu} j_{s}^{3}+F_{\mu \nu} \mathcal{G}_{3}^{\mu \nu} j_{p}+F_{\mu \nu} \mathcal{G}^{\mu \nu} j_{p}^{3}\right. \\
\left.+\frac{1}{3}\left(F_{\mu \nu} \mathcal{G}^{\mu \nu} j_{p}+F_{\mu \nu} \mathcal{F}^{\mu \nu} j_{s}+F_{\mu \nu} \mathcal{G}_{i}^{\mu \nu} j_{p}^{i}+F_{\mu \nu} \mathcal{F}_{i}^{\mu \nu} j_{s}^{i}\right)\right], \\
I_{V A}=2 i \epsilon_{i j 3}\left(g_{2} A_{\rho} V_{i}^{\rho} j_{s}^{j}+g_{2 A} A_{\rho} \bar{A}_{i}^{\rho} j_{p}^{j}\right), \\
I_{V A m}=g_{2}\left[A^{\nu} V_{\nu}^{3} j_{s}+A^{\nu} V_{\nu} j_{s}^{3}\right]+g_{2 A}\left[A^{\rho} \bar{A}_{\rho}^{3} j_{p}+A^{\mu} \bar{A}_{\mu} j_{p}^{3}\right] \\
+\frac{g_{2}}{3}\left[A^{\nu} V_{\nu} j_{S}+A_{\rho} V_{i}^{\rho} j_{S}^{i}\right]+\frac{g_{2 A}}{3}\left[A^{\mu} \bar{A}_{\mu} j_{p}+A^{\mu} \bar{A}_{\mu}^{i} j_{p}^{i}\right], \\
I_{V F}=i \epsilon_{i j 3}\left(g_{3} F_{\rho \delta} V_{i}^{\rho} j_{j, V}^{\delta}+g_{3 A} F_{\rho \delta} \bar{A}_{i}^{\rho} j_{j, A}^{\delta}\right), \\
\left.I_{A F}=i \epsilon_{i j 3}\left(g_{3} \mathcal{F}_{\rho \delta}^{i} A^{\rho} j_{j, V}^{\delta}+g_{3 A} \mathcal{G}_{\rho \delta}^{i} A^{\rho} j_{j, A}^{\delta}\right)\right),
\end{gathered}
$$

where the effective coupling constants have been defined in Euclidean momentum space as

$$
\begin{gathered}
g_{1}=N_{c} d_{1} e M^{*}\left(\alpha g^{2}\right) \operatorname{Tr}^{\prime}\left(\left(\tilde{S}_{0}(k) \tilde{S}_{0}(k) \tilde{S}_{0}(k) R(k)\right)\right), \\
g_{2}=6 N_{c} d_{1} e M^{*}\left(\alpha g^{2}\right) \operatorname{Tr}^{\prime}\left(\left(\tilde{S}_{0}(k) \tilde{S}_{2}(k) R(k)\right)\right), \\
g_{2 A}=6 N_{c} d_{1} e M^{*}\left(\alpha g^{2}\right) \operatorname{Tr}^{\prime}\left(\left(\tilde{S}_{0}(k) \tilde{S}_{2 A}(k) R(k)\right)\right), \\
g_{3}=\frac{g_{2}}{M^{*}}, \quad g_{3 A}=\frac{g_{2 A}}{M^{*}},
\end{gathered}
$$

where 


$$
\tilde{S}_{0}(k)=\frac{1}{k^{2}+M^{* 2}}, \quad \tilde{S}_{2}(k)=\frac{k^{2}-M^{* 2}}{\left(k^{2}+M^{* 2}\right)^{2}}, \quad \tilde{S}_{2 A}(k)=\frac{k^{2}+M^{* 2}}{\left(k^{2}+M^{* 2}\right)^{2}}
$$

Therefore the leading axial mesons-photon couplings to constituent quarks axial currents are not exactly the same as the corresponding chiral partners vector mesons-photons couplings to constituent quarks vector currents and photons.

The following $B_{0}$-dependent anisotropic vector mesons-constituent quark couplings emerge:

$$
\begin{gathered}
I_{F F} \rightarrow 2 g_{1}^{B} i \epsilon_{i j 3}\left[\mathcal{G}_{i}^{x y} j_{p}^{j}+\mathcal{F}_{i}^{x y} j_{s}^{j}\right], \\
I_{F F m} \rightarrow g_{1}^{B}\left[\mathcal{F}_{3}^{x y} j_{s}+\mathcal{F}^{x y} j_{s}^{3}+\mathcal{G}_{3}^{x y} j_{p}+\mathcal{G}^{\mu \nu} j_{p}^{3}+\frac{1}{3}\left(\mathcal{G}^{x y} j_{p}+\mathcal{F}^{x y} j_{s}+\mathcal{G}_{i}^{x y} j_{p}^{i}+\mathcal{F}_{i}^{x y} j_{s}^{i}\right)\right], \\
I_{V A} \rightarrow 2 i \epsilon_{i j 3}\left[g_{2 A}^{B} \bar{A}_{i}^{y} j_{p}^{j}+g_{2}^{B} V_{i}^{y} j_{S}^{j}\right], \\
I_{V A m} \rightarrow g_{2}^{B}\left[V_{y}^{3} j_{s}+V_{y} j_{S}^{3}\right]+g_{2 A}^{B}\left[\bar{A}_{y}^{3} j_{p}+\bar{A}_{y} j_{p}^{3}\right]+\frac{g_{2}^{B}}{3}\left(V_{y} j_{S}+V_{i}^{y} j_{S}^{i}\right)+\frac{g_{2 A}^{B}}{3}\left(\bar{A}_{y} j_{p}+\bar{A}_{y}^{i} j_{p}^{i}\right), \\
I_{V F} \rightarrow i \epsilon_{i j 3}\left[g_{3}^{B_{1}} V_{i}^{x} j_{j, V}^{y}+g_{3 A}^{B_{1}} \bar{A}_{i}^{x} j_{j, A}^{y}\right], \\
\left.I_{A F} \rightarrow i \epsilon_{i j 3}\left[g_{3}^{B_{2}} \mathcal{F}_{y \delta}^{i} j_{j, V}^{\delta}+g_{3 A}^{B_{2}} \mathcal{G}_{y \delta}^{i} j_{j, A}^{\delta}\right)\right],
\end{gathered}
$$

where the effective coupling constants have been defined in the long wavelength limit,

$$
\begin{gathered}
g_{1}^{B}=6 N_{c} d_{1} M^{*}\left(e B_{0}\right)\left(\alpha g^{2}\right) \operatorname{Tr}^{\prime}\left(\left(\tilde{S}_{0}(k) \tilde{S}_{0}(k) \tilde{S}_{0}(k) R(k)\right)\right), \\
g_{2}^{B}=6 N_{c} d_{1}\left(e B_{0}\right) M^{*}\left(\alpha g^{2}\right) \operatorname{Tr}^{\prime}\left(\left(-\tilde{S}_{0}(k) \frac{\partial}{\partial q_{x}} S_{2}(k ; k+q) R(k)\right)\right)_{q=0}, \\
g_{2 A}^{B}=6 N_{c} d_{1}\left(e B_{0}\right) M^{*}\left(\alpha g^{2}\right) \operatorname{Tr}^{\prime}\left(\left(-\tilde{S}_{0}(k) \frac{\partial}{\partial q_{x}} S_{2 A}(k ; k+q) R(k)\right)\right)_{q=0} \\
g_{3}^{B_{1}}=6 N_{c} d_{1}\left(e B_{0}\right)\left(\alpha g^{2}\right) \operatorname{Tr}^{\prime}\left(\left(\tilde{S}_{0}(k) \tilde{S}_{2}(k) R(k)\right)\right), \\
g_{3 A}^{B_{1}}=6 N_{c} d_{1}\left(e B_{0}\right)\left(\alpha g^{2}\right) \operatorname{Tr}^{\prime}\left(\left(\tilde{S}_{0}(k) \tilde{S}_{2 A}(k) R(k)\right)\right), \\
g_{3}^{B_{2}}=6 N_{c} d_{1}\left(e B_{0}\right)\left(\alpha g^{2}\right) \operatorname{Tr}^{\prime}\left(\left(-\tilde{S}_{0}(k) R(k) \frac{\partial}{\partial q_{x}} \tilde{S}_{2}(k ; k+q)\right)\right)_{q=0} \\
g_{3 A}^{B_{2}}=6 N_{c} d_{1}\left(e B_{0}\right)\left(\alpha g^{2}\right) \operatorname{Tr}^{\prime}\left(\left(-\tilde{S}_{0}(k) R(k) \frac{\partial}{\partial q_{x}} \tilde{S}_{2 A}(k ; k+q)\right)\right)_{q=0},
\end{gathered}
$$

where the functions were defined as

$$
\tilde{S}_{2}(k ; k+q)=\frac{k \cdot(k+q)-M^{* 2}}{\left(k^{2}+M^{* 2}\right)\left((k+q)^{2}+M^{* 2}\right)}, \quad \tilde{S}_{2 A}(k ; k+q)=\frac{k \cdot(k+q)+M^{* 2}}{\left(k^{2}+M^{* 2}\right)\left((k+q)^{2}+M^{* 2}\right)}, \quad \tilde{S}_{0}(k)=\frac{1}{k^{2}+M^{* 2}} .
$$

The corresponding gauge-type coupling constants of vector and axial mesons to constituent quarks in the vacuum as presented in Refs. [1,2] are correctly written as 


$$
L_{v-q}=g_{r 1} V_{i}^{\mu}(x) j_{\mu}^{V, i}(x)+g_{a 1} \bar{A}_{i}^{\mu}(x) j_{\mu}^{A, i}(x)+g_{v 1} V^{\mu}(x) j_{\mu}(x)+g_{f 1} \bar{A}_{\mu}(x) j_{A}^{\mu}(x),
$$

where the dimensionless coupling constants were found to be

$$
\begin{aligned}
& g_{r 1}=g_{v 1}=4 i d_{1} N_{c}\left(\alpha g^{2}\right) \operatorname{Tr}^{\prime}\left(\left(\bar{S}_{2}(k) \overline{\bar{R}}(k)\right)\right), \\
& g_{a 1}=g_{f 1}=4 i d_{1} N_{c}\left(\alpha g^{2}\right) \operatorname{Tr}^{\prime}\left(\left(\bar{S}_{2 A}(k) \overline{\bar{R}}(k)\right)\right) .
\end{aligned}
$$

F. L. B. participates in the project INCT-FNA, Proc. 464898/2014-5, and acknowledges partial support from Grants No. CNPq/312072/2018-0 and No. CNPq/421480/2018-1.

[1] F. L. Braghin, Light vector and axial mesons effective couplings to constituent quarks, Phys. Rev. D 97, 054025 (2018).

[2] F. L. Braghin, Phys. Rev. D 101, 039901 (2020). 